\title{
Chronik
}

\section{„Hôpitaux en France, en Allemagne et en Italie. Une histoire comparée (Moyen Âge et Temps Modernes). - Hospitäler in Frankreich, Deutschland und Italien. Eine vergleichende Geschichte (Mittelalter und Neuzeit) ${ }^{\star}$}

Am 19. September 2003 fand unter der organisatorischen Verantwortung von Gisela Drossbach in Zusammenarbeit mit dem Deutschen Historischen Institut Paris, dort Herm Professor Paravicini, und mit Unterstützung der Gerda Henkel Stiftung ein intermationales Kolloquium unter dem Titel .. Hopitaux en France. en Allemagne et en Italie. Une histoire comparée (Moven Age et Temps Modernes). - Hospitäler in Frankreich. Deutschland und Italien. Eine vergleichende Geschichte (Mittelalter und Neuzeit) " statt. Damit wurde ein in der gegenwärtigen mediävistischen und frühneuzeitlichen Forschung mit großem Interesse wahrgenommenes Thema behandelt (siehe auch zuletzt die Tagungen von Alzey, Reichenau, Amiens. S. Miniato). Bei diesem Kolloquium wurde insbesondere die Komplexität und Vielschichtigkeit des Hospitals erarbeitet, indem auf ausgewählte institutionelle Aspekte näher eingegangen wurde.

In seiner Begrüßung wies Werner Paravicini auf die Aktualität des Themas hin, die in vielen neuen Publikationen zum Ausdruck kommt. Erwähnt wurden auch die zahlreichen Bibliotheken und Archive insbesondere in Frankreich, die ausschließlich Quellen und Literatur zur Geschichte des Hospitals aufbewahren. Eine von drei solchen Einrichtungen in Paris sind die „Archives de l'Assistance Publique - Hôpitaux de Paris", der ein Besuch während des Kolloquiums gewidmet war.

In einer Hinfuihrung zum Thema wurde der Forschungsstand zu Frankreich (Francoise-Olivier Touati), Deutschland (Gisela Drossbach) und Italien (Thomas Frank) in seinen großen Linien verglichen. Bis in die 60er Jahre des 20. Jahrhunderts dominierte in der deutschen Spitalforschung, vorgezeichnet durch den Rechtshistoriker Siegfried Reicke, ein verfassungsgeschichtlicher Ansatz, während in Frankreich und inshesondere in Italien das mittelalterliche Hospital als Element der Kirchen- und Ordensgeschichte gesehen wurde. Ein Wandel trat zu Beginn der 70er Jahre durch Arbeiten wie die von Michel Mollat und Charles de La Roncière ein, die sich den Themen Armut, Arme und Armensorge aus sozialgeschichtlicher Sicht zuwandten. „Assistenza“ anstelle von „caritas“ wurde der Leitbegriff der sozial- und wirtschaftsgeschichtlich geprägten italienischen Forschung. Ab den 80er Jahren wurden sodann eine Vielzahl unterschiedlicher Forschungsansätze und Fragestellungen auch auf das Thema des Hospitals angewandt, wie beispielsweise prosopographische Studien, Arbeiten über die „Sozialdisziplinierung“ zwischen Armensorge und Armenkontrolle, Funktionalität und Funktionswandel etc. Am Vorabend der Tagung des 
Konstanzer Arbeitskreises für Geschichte im Frühjahr 2002, der sich das Thema der Sozialgeschichte des mittelalterlichen Hospitals gewählt hatte, stand fest: „das Hospital" hat es im Mittelalter nicht gegeben-Zitat Karl-Heinz Spieß: ,Zu unterschiedlich sind die Insassen der Hospitäler [...], unterschiedlich auch die Träger [...], und schließlich die Funktionen (das Hospital als Altersheim, als Schule wie in Nürnberg. als Geldinstitut oder geselliger Treffpunkt, wie es bei dem Wiener Bürgerspital mit seinen Wein- und Bierkellem nachgewiesen wurde) ${ }^{* 1}$ ). In unmittelbarer Anbindung und Fortsetzung dieser ergebnisreichen Tagung bot es sich nun an, die Heterogenität der Hospitäler emeut wahrzunehmen und in ihrer Vielfalt zu erforschen. Dies betraf im Detail: Quellen, Finanzen, Versorgung, Frauen und Männer, Memoria.

Die Quellenfrage ist schon deshalb von Bedeutung, da es nicht nur die Möglichkeiten. sondern auch die Grenzen der Erforschung von Hospitälern deutlich macht. Denn Größe und Bedeutung des mittelalterlichen Hospitals sind unter anderem dafür ausschlaggebend, dass heute überhaupt noch Archivalien erhalten sind, die weitere Forschungen möglich machen. Zwei Beiträge beschäftigten sich mit den Quellen zum Hospital. Der eine war den archivalischen Beständen zum Hospital gewidmet, der andere der Normativität von Hospitalstatuten.

Beate Sophie Fleck/Münster (,Zur Quellenlage westfälischer Hospitäler im 15. und 16. Jahrhundert am Beispiel von Hospitalinsassen") konnte aufgrund ihrer fundierten Kenntnisse der Archive in Westfalen die verschiedenen Quellenbestände von Hospitälern nachweisen, die sowohl Hospitalbestand sein konnten wie auch in Archiven außerhalb eines Hospitals, beispielsweise Stadtarchiven, aufbewahrt wurden. Diese archivalische Situation verdeutichte sie am Beispiel der Hospitalinsassen, zu welchen sie Auskunft über deren unterschiedliche Personenkreise, Aufnahmepraxen, Nahrungsgewohnheiten etc. gab. Nach der Einschätzung von Sophie Fleck ist diese Form des Quellenbestandes auch in anderen Regionen Deutschlands vorzufinden.

Gisela Drossbach/München (,Genese, normative Strukturen und Funktionen von Hospitalstatuten: Frankreich, Deutschland und Italien im Vergleich“) unternahm den Versuch, die komplexen Bestimmungen für ein Hospital, wie sie als Regeln (regulae), Statuten (statuta) oder Ordnungen (,ordonnances") vorzufinden sind, als literarische Gattung vorzustellen. Einige dieser exemplarisch ausgewählten, sogenannten Spitalstatuten untersuchte sie auf ihre inhaltlichen Schwerpunkte, wie Normen des Zusammenlebens der den Spitaldienst Leistenden sowie der Spitalinsassen, die von der Spitalbruderschaft zu erbringenden geistlichen Leistungen und die Organisations- und Verfassungsstruktur des Spitals. Leitfrage war hierbei, inwieferm Spitalstatuten das ,ganze Hospital“ widerspiegeln, $d$. h. auf die institutionelle und auch verfassungsmäßige Wirklichkeit des Hospitals eingehen oder eben nur Teilbereiche des Hospitals ansprechen.

Unter den Finanzen des Spitals stellt man sich in der Regel Berichte zu umfangreichen Rechnungsbüchern mit Statistiken vor. Ganz anderer Art waren die folgenden zwei Beiträge zum Thema. Andreas Meyer/Marburg („Beobachtungen zu den Finanztransaktionen des Hospitals von Altopascio im 13. Jahrhundert") beschäftigte sich mit dem Sankt-Jakob Hospital von Altopascio/Toscana, das bereits seit der zwei-

') Protokoll Nr. 387 über die Arbeitstagung auf der Insel Reichenau vom 19.-22. März 2002, Thema: „Sozialgeschichte mittelalterlicher Hospitäler“, S. 3. 
ten Hälfte des 11. Jahrhunderts bestand und Pilgern, Armen sowie Kranken diente. Dabei fiel der plötzliche Reichtum des Hospitals um 1200 auf, als dessen Ursache Andreas Meyer die weitreichenden Sammellizenzen des Hospitals erkennen konnte. Wie aber gelangten die eingesammelten Gelder aus den verschiedenen Ländern und Regionen nach Altopascio? Anhand unedierter Quellen konnte Andreas Meyer belegen, dass das Hospital für seine Sammelerträge die Dienste der Kaufleute in Anspruch nahm. die das Geld vom Erwerbsort an die Zentrale transferierten. In Weiterentwicklung dieses Systems ist das Finanzwesen des Hospitals von Altopascio möglicherweise als Vorbild für die wenig später entstandene Papstfinanz zu werten.

Aus anderer Perspektive, nämlich in den Kategorien von Repräsentation und Memoria, beleuchtete Julien Dema de/Göttingen (,Compter pour Dieu et les pauvres. La gestion de l'hôpital de Nuremberg comme justification lä̈que dans le champ ecclésiastique, 1339-1525") das Finanzwesen des Nümberger Heilig-Geist-Spitals. Bereits mit seinem Stiftungsbrief von 1339 initiierte der Nürnberger Kaufmann und Patrizier Konrad Groß eine umfangreiche Dokumentation von Verwaltung und Finanzen. Nach Julien Demade stand die aufwendige, in der Schreibstube repräsentativ aufbewahre Rechnungslegung im Dienste der memoria für den Stifter und sollte Dauerhaftigkeit (durabilité) symbolisieren. Im 15. Jahrhundert ging die Verwaltung des Hospitals an den Rat über, der das Finanzmodell auf klösterliche Einrichtungen übertrug und diese in der Folgezeit erfolgreich kontrollierte.

Dem Thema der Emährung und Versorgung der armen Kranken sowie des Personals im Pariser Hôtel-Dieu widmete sich Christine Jehanno/Paris (,.Sustenter les ,povres malades“. Alimentation et approvisionnement à la fin du Moyen Âge: l'exemple de l'Hôtel-Dieu de Paris") auf der Basis der Spitalstatuten aus dem Jahre 1535. Dabei stellte Christine Jehanno fest, dass die Ernährung nicht auf die spezifische Krankheit der Patienten abgestimmt und damit integraler Teil der Krankenpflege war. Vielmehr orientierte sich der Speiseplan an der Hierarchie des Hospitalpersonals: oben rangierten der Meister und der Prior, gefolgt von den Brüdern und Schwestern, dann erst die bediensteten Laien und die Kranken. Das heißt, die Regel gab die Ernährungspraktiken der einfacheren Pariser Bevölkerungsschichten wieder. Letztlich musste offen bleiben, ob die Patienten nicht wirklich krank, sondern eher schwach waren und ihnen eine einfache Kost genügte, oder ob der Speiseplan nur Theorie war.

Zum Schweigen der Quellen bezüglich der medizinischen Versorgung in den Quellen des Früh- und Hochmittelalters bot Francois-Olivier Touati/Paris (.,Médecins et soins médicaux dans les hôpitaux au Moyen Âge (Orient-Occident) pour une réévaluation") eine eigene Lösung.

Aus sozialgeschichtlicher Sicht untersuchte der Experte für Florentiner Hospitäler der Renaissance, John Henderson/Cambridge („Caring for the Poor: Commessi and Commesse in the hospitals of medieval and renaissance Florence") die Personengruppe der "commesse, oblate, dedicate". Dabei griff er auf bisher unveröffentlichtes Quellenmaterial zurück, nämlich auf die Verträge, welche jene Personen eingingen, die ins Hospital aufgenommen werden wollten. Diese Verträge konnten Aufschluss über die Gründe für den Eintritt ins Hospital geben. Da die aufzunehmenden Frauen fast alle „Singles“, also unverheiratete Frauen oder Witwen waren, schloss Henderson daraus, dass der Wunsch der Frauen um Aufnahme als commesse nicht immer freiwillig und aus Motiven religiöser Hingabe erfolgte, sondern von wesentlicher Bedeutung für ihr Überleben war. 
Das komplexe Thema der Memoria wurde aus drei sehr verschiedenen Perspektiven angegangen: der kunsthistorischen, der kirchengeschichtlichen und der prosopographischen. Durch Brigitte Kurmann-Schwarz/Zürich (,Les commanditaires et leurs volontés: l'exemple de l'hôpital de Tonnerre et de sa Mise au tombeau") erfuhr das Hospital in Tonnerre erstmals eine eingehende kunsthistorische Untersuchung. Margarete von Burgund, Witwe Karls I. von Anjou, Königs von Jerusalem und Sizilien. hatte das Hospital 1293 gegründet. Obwohl nur sie ihre Grablege dort haben würde, bezeichnet sie in ihrer Stiftungsurkunde Karl I. von Anjou als Gründer des Hospitals und bezog ihn in die zu leistenden Gebetsgottesdienste mit ein. So belegte die Rekonstruktion der visuellen Quellen nicht nur das Hospital als einen Ort der Memoria für Margarete und ihren königlichen Gatten, sondern es darf künftig auch zu den Vorbildern der Gründung des Hôtel d'Dieu in Beaune durch Nicolas Rolin gezählt werden.

An die Bedeutung des Hospitals als Ort der Grablege schloss sich trefflich der Beitrag von Thomas Frank/Rom-Berlin (,Die Sorge um das Seelenheil in italienischen, deutschen und französischen Hospitälem") an. Er konnte feststellen, dass die Verbindung von caritas und memoria bereits im antiken Christentum eine bedeutende Rolle spielte und die Theologie des Mittelalters den sechs biblischen Werken der Barmherzigkeit eine Tat hinzufügte: die Bestattung der Toten. Auf diese Weise wurde die memoria ein Teil der caritas. Des weiteren konnte Thomas Frank aufzeigen, dass die Memoria nicht nur dem Stifter galt, sondern dass auch die Armen daran partizipieren konnten, sodass insgesamt eine Vielzahl von Personen und Personengruppen daran beteiligt waren. Die von Thomas Frank notwendigerweise getroffene Einschränkung auf die liturgische Memoria war auch als Anregung zur Erforschung weiterer in mittelalterlichen Hospitälern üblichen Verfahren zur Förderung des Seelenheils gedacht.

Auf andere Weise würdigte Andreas Rehberg/Rom (,Die Römer und ihre Hospitäler. Überlegungen zu den Motiven der Hospitalsgründungen in Rom, 13.-15. Jh.“) das Thema caritas et memoria. In Antwort auf die Frage nach Absicht und Zweck von Hospitalgründungen in Rom konnte erstmalig die Unterscheidung von verschiedenen „Hospitaltypen“ im spätmittelalterlichen Rom getroffen werden. Bezüglich der Einteilungskriterien konnte Andreas Rehberg auf sein Wissen um die dortige prosopographische Situation zurückgreifen. Darauf basierend unterschied er vier „Typen“: das Hospital der Päpste (Hospital von S. Spirito in Sassia), das Krankenhospiz unter der Protektion römischer Bürger (Hospital von S. Giovanni in Laterano), das Hospital römischer Bruderschaften (Hospital von S. Maria in Portico), das landsmannschaftliche Hospital (Hospital der Anima).

Nachdem die institutionelle Vielfalt des mittelalterlichen und frühneuzeitlichen Hospitals in Frankreich, Deutschland und Italien herausgestellt worden war, konzentrierte sich die Schlussdiskussion darauf, von der Vielheit wieder zur Einheit zu gelangen - gab es ,das Hospital“ doch?

Weitere Aufschlüsse und Ergebnisse wird der Tagungsband geben, der in der Reihe „Pariser Historische Studien“ erscheinen wird ${ }^{2}$ ).

München

Gisela Drossbach

2) Der hier vorliegende Text wird auch als Information der Zeitschrift Außeruniversitären Historischen Forschung AHF im Internet veröffentlicht. 


\section{ZRG-RICHTLINIEN FÜR DIE MANUSKRIPTGESTALTUNG}

Es können nur inhaltlich, orthographisch und grammatikalisch druckreife, gut lesbare Manuskripte angenommen werden. Änderungen gegenüber dem Manuskript sind im Korrekturgang nur in sehr beschränktem Umfang ausnahmsweise durchführbar. Disketten sind zusätzlich zum Papierabzug willkommen. Die eingereichten Manuskripte verbleiben beim Verlag, außer auf Anfrage.

Manuskriptseiten bitte einseitig locker beschreiben.

Haupttext: Lateinische Wörter und Texte sind kursiv zu schreiben oder durch Unterwellen hervorzuheben, wobei Anführungszeichen entfallen (Ausnahme: längere Quellenzitate als Absatz, petit). Fremde Schriften und Schriftzeichen müssen deutlich und korrekt geschrieben sein, am besten mit einer entsprechenden Schreibmaschine, oder in einer guten Photokopie ins Manuskript eingeklebt werden. Bitte weisen Sie bei elektronischer Datenübertragung extra auf Sonderzeichen hin. Fußnotenverweisziffern stehen immer vor der Interpunktion.

Absätze und Leerzeilen müssen deutlich erkennbar sein, auch an Seitenübergängen.

Fußnoten beginnen mit Großbuchstaben und enden mit Schlusspunkten. Namen (samt Vornamen) modemer Autoren sind gesperrt zu schreiben oder zu unterstreichen. Latein ist wie im Haupttext zu markieren. Buch- und Aufsatztitel werden nicht hervorgehoben, auch nicht durch Anführungszeichen. Titel von Aufsätzen und Erscheinungsorte können bei leicht auffindbaren Titeln entfallen. Der Verweis auf Schriftenreihen in den Fußnoten ist unerwünscht. Das Erscheinungsjahr ist in jedem Langzitat anzugeben.

Statt Leerverweisen (a. a. O., l. c.) ist auf das vorangegangene Langzitat (o. Fn. •) oder mit Kurztitel zu verweisen. Klammern in Klammern vermeiden.

Diese Zeitschrift wird zitiert: ZRG 100 Kan. Abt. 69 (1983) 1 beziehungsweise ZRG Germ. Abt. 100 (1983) 1; ZRG Rom. Abt. 100 (1983) 1 = SZ 100 (1983) 1; Digesten: D. 1,1,1 pr.

Abkürzungen: n. Chr., ff., S., Anm.. Bd., Lit., St., u. s. w., z. B., vgl., etc. sind allgemein üblich, auf andere bitte verzichten. Siglen müssen am Anfang eines Beitrages definiert werden.

Bucbbesprechungen müssen im Kopf alle bibliographischen Angaben wiedergeben, auch Reihentitel, Verlag, Verlagsort, Erscheinungsjahr und Umfang.

Es gibt nur einen Korrekturgang. Die Korrekturabzüge weisen die endgültige Seitenzählung auf. Der Korrekturgang dient primär nur noch der Kontrolle des Setzers. Änderungen gegenüber dem Manuskript im gesetzten Text sind zu vermeiden oder auf das Mindestmaß zu beschränken. Umbruchändernde Autorkorrekturen werden nicht ausgeführt. Außerordentliche Autorkorrekturen können dem Autor vom Verlag in Rechnung gestellt werden.

Lektorat: DDr. Reingard Rauch, Waldheimatweg 33, A-8043 Graz +443-316-32 33 83, 3382 40, reingard.rauch@gmx.at 

